\title{
Geomagnetic Storms and Substorms as Space Weather Influence on Atmospheric Electric Field Variations
}

\author{
N.G. Kleimenova ${ }^{1}$, A. Odzimek ${ }^{2}$, S. Michnowski ${ }^{2}$, M. Kubicki ${ }^{2}$ \\ ${ }^{1}$ Schmidt Institute of Physics of the Earth RAS, Moscow, Russia \\ ${ }^{2}$ Institute of Geophysics PAS, Warsaw, Poland \\ E mail (kleimen@ifz.ru).
}

Accepted: 12 October 2018

\begin{abstract}
The atmospheric electric field (Ez) exists in the Earth's atmosphere at any location on the globe due the phenomenon called the global atmospheric electric circuit. This global circuit is controlled mainly by the world thunderstorm and shower clouds activity which act as generators, and the cosmic rays as well solar electromagnetic radiation, which modify its load. Moreover, the Earth's magnetosphere-ionosphere disturbances, caused by interaction with solar wind, could influence this state as well. Here we present an overview of our main results of the study of the potential effects in Ez caused by geomagnetic storms and substorms as an important factor of the space weather. Our results are based of the Ez observations at the mid-latitude Polish station Swider (near Warsaw) and high-latitude Polish station Horsund (Spitsbergen archipelago). The effect of magnetic storms, associated with a coronal mass ejection from the Sun, was detected in the mid-latitude atmospheric electricity as strong daytime Ez negative anomalies in relation with simultaneous occurrence of night-side magnetospheric substorms. However, at high-latitudes, the auroral-latitude magnetic substorm lead to the simultaneous polar-latitude Ez deviations, positive in the local morning and negative in the evening, corresponding to the station's location relative to the "positive" or "negative" center of the polar ionosphere convection vortex. Thus, ground-based Ez recordings could be one of the very sensible tools to study solar windmagnetosphere-ionosphere-atmosphere interactions.
\end{abstract}

(c) 2018 BBSCS RN SWS. All rights reserved

Keywords: atmospheric electricity, electric field, magnetosphere, geomagnetic substorm, magnetic storm, space weather, solar wind, interplanetary magnetic field

\section{Introduction}

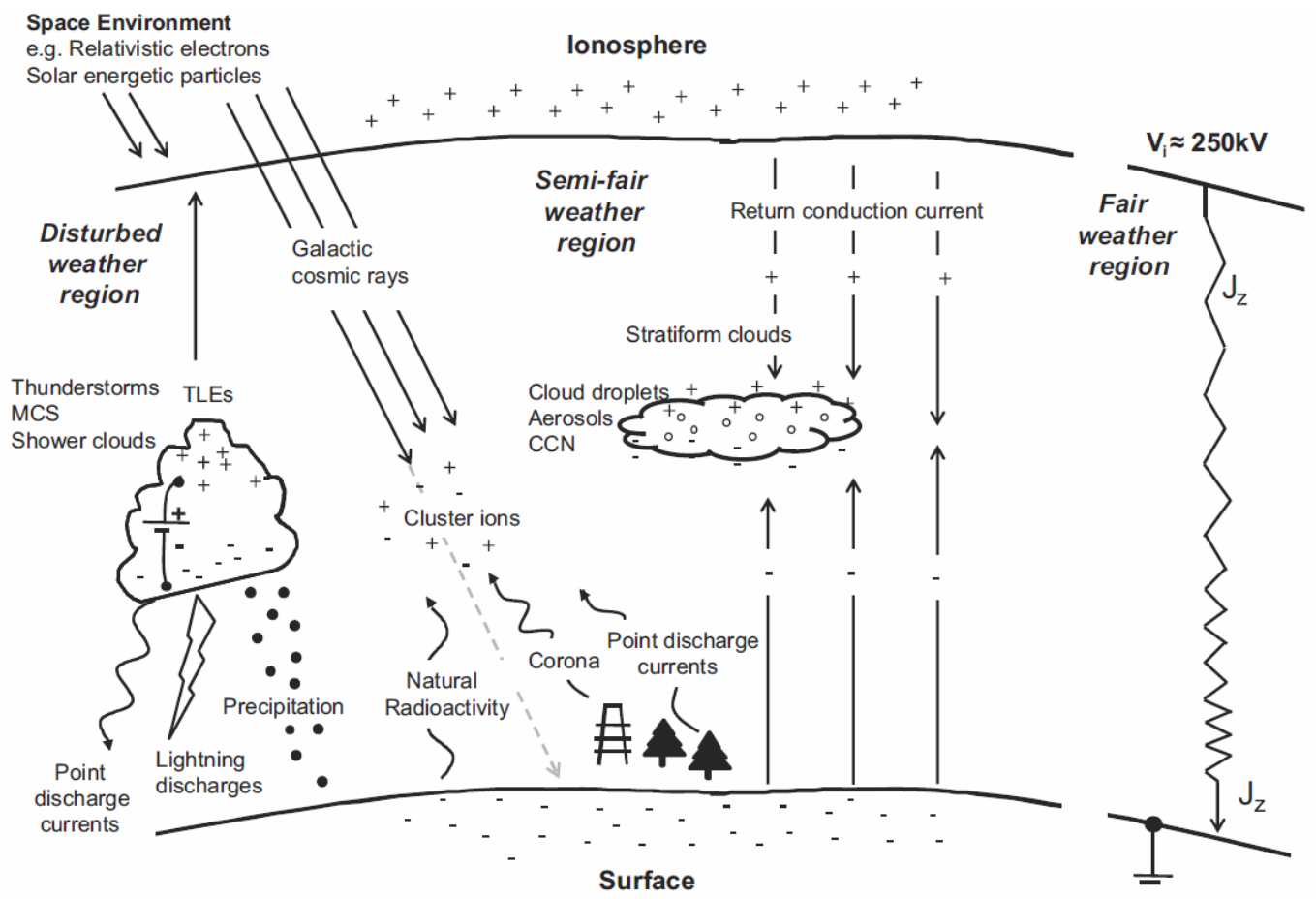

Fig. 1. Schema of the Earth's global atmospheric electric circuit (after Rycroft et al. 2012)

Atmospheric electricity is one of the important geophysical topics. More than one hundred years ago it was discovered that the atmospheric electric field exists near the Earth surface even in the absence of local thunderstorm activity. The concept of the global atmospheric electric circuit (GEC) was formulated by Wilson (1920). The global circuit is formed between the surface of the Earth and the ionosphere, both of which are good electrical conductors in comparison with the weakly conducting atmosphere. General features of the Earth's global circuit are shown in the schematic diagram by Rycroft et al. (2012), here reproduced in Fig. 1. 
The atmosphere is ionised by cosmic rays and the ionisation is modulated solar energetic particles. Additional ionization of the air near the Earth's surface comes from natural radioactivity over land. The ionised atmosphere maintains the circuit's air-Earth electric current, flowing in fair-weather region due to the potential difference between the Earth's surface and the ionosphere which is charged positively relative to the Earth's surface (e.g., Tinsley, 2000).

The ionosphere is considered an equipotential surface except at high geomagnetic latitudes where it also participates in the current system of the Earth's magnetosphere resulting from its interaction with the solar wind. In addition to the air-Earth current, the vertical atmospheric electric field (Ez) of $\sim 100 \mathrm{~V} / \mathrm{m}$ in fair weather at ground level, greater than the horizontal components of the field because of the main direction of the current flow in the GEC.

The topic of the global atmospheric electric circuit has been discussed and reviewed last decades by many authors (e.g., Rycroft and Harrison, 2011; Williams and Mareev, 2014). They presented the background of the subject and gave a large number of key references to the literature. Despite being postulated almost hundred years ago, the global electric circuit is still poorly quantified (Tinsley and Zhou 2006; Odzimek and Lester, 2009).

As the global atmospheric electric circuit is closed through the Earth's ionosphere, it can be affected by magnetospheric and ionospheric disturbances. The physical background of plausible solar wind influences on atmospheric electricity variables in the highly coupled system representing the Earth's atmosphere and the near-Earth space environment has been discussed by Michnowski (1998). The ground-based observations at the auroral latitudes have indicated such effects (e.g., Sao, 1967; Olson, 1971; Zhdanov et al., 1984; Roble, 1985; Bandilet et al., 1986; Michnowski et al., 1991, 1996; Belova, 2000). It was found that the nigh-side magnetic substorm can change the Ez values. The influence of the Interplanetary Magnetic Field (IMF), magnetosphere-ionosphere disturbances and polar ionospheric convection on the groundbased Ez variations at the polar latitudes has been found in polar cap area Antarctica (e.g., Tinsley, 2000; Frank-Kamenetsky et al., 2001, 2012) as well as in the Arctic (Odzimek et al. , 2011).

The most important factors of the space weather state in the the Earth's environment are magnetic storms (e.g., Gonzales et al. 1994) and substorms (e.g., Akasofu, 1964). The ionosphere disturbances, caused by magnetic storm and substorm development, could change the state of the global atmospheric electric circuit, what should appear in the variations of the ground-based Ez values.

The aim of this paper is to pay attention on some potential influence of the main factor of cosmic weather, such as geomagnetic storms and substorms, on variations of the atmospheric electric field Ez, and overview our most important results obtained during the last 15 years in the Ez investigations at high and low latitudes (Nikiforova et al., 2003, 2005; Kleimenova. et al., 2008, 2010, 2011, 2013, 2017; Michnowski et al. 1996, 2007, 2014; Kubicki et al., 2014). In this paper, we did not intend to conduct serious statistical studies. We presented here only some examples of possible effects of magnetic storms and substorms on temporal variations of the Ez measured at the middle and high latitudes.

\section{Diurnal global variations of the atmospheric electric field}

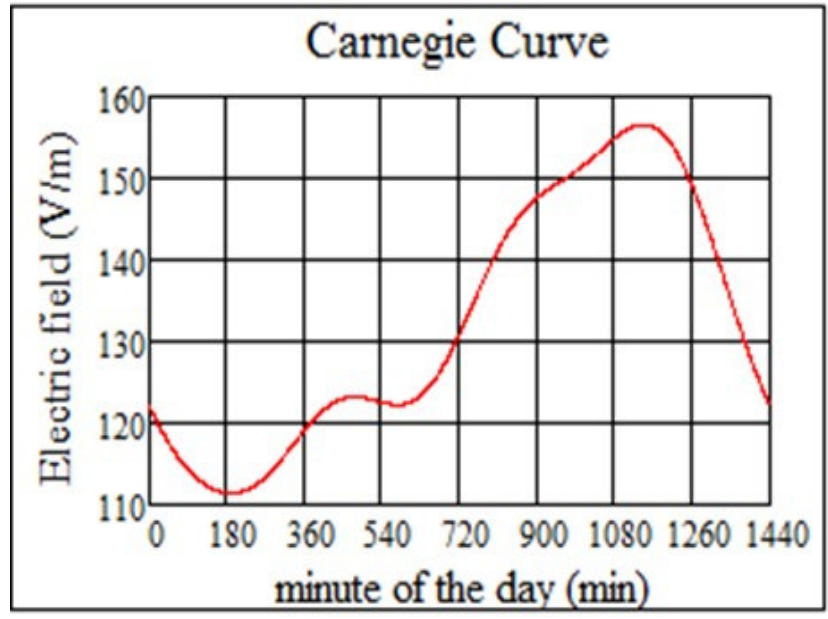

Fig. 2. The diurnal global Ez variation - Carnegie curve.

The atmospheric electric field near the ground level is usually influenced by many meteorological factors and thus locally varies from its global character. Only in so called atmospheric electricity "fair weather" conditions, defined by the absence of precipitation or fog, high wind speed and absence of high or low cloudiness it is believed to reflect the global signal. Pollution (aerosols) also affects it and therefore the most reliable observations of the GEC should be made in places where the weather conditions are stable and pollution is low.

An important result in the studies of the global circuit has been obtained by the measurements of the atmospheric electric field in the clean air at the Atlantic, Indian, and Pacific oceans during four global cruises of the wooden non-magnetic vessel named "Carnegie" in the first half of the 20th century (Parkinson and Torreson, 1931). The diurnal average variation of Ez during "fair weather" conditions calculated from measurements from "Carnegie" demonstrates a variation in universal time (UT) with a minimum around 03-04 UT and a maximum around 1821 UT, and is known as the Carnegie curve (Fig. 2). The character of the variation is believed to originate mainly from the regular daily variations in the world electrified cloud activity concentrated mainly in three centres in Asia, Africa, and America. Observations of the atmospheric electric field over the globe confirm this result to some extent (e.g., Kubicki et al. 2016).

\section{Observations and results}

Results summarised here are based on the data of the vertical component of the atmospheric electric field (Ez), observed at two ground-based stations: the mid-latitude Polish Geophysical Observatory Swider located near Warsaw (SWI, corrected geomagnetic coordinates: $\left.\Phi^{\prime}=47.81^{\circ}, \Lambda^{\prime}=96.81^{\circ}\right)$ and the polarlatitude Polish Station Hornsund located in Spitsbergen archipelago (HOR, corrected geomagnetic coordinates: $\left.\Phi^{\prime}=74.01^{\circ}, \Lambda^{\prime}=110.51^{\circ}\right)$. The electric field 
recording equipment is described in more detail in Kubicki (2001) and Berlinski et al. (2007). The average diurnal variations of $\mathrm{Ez}$ at Swider and Hornsund are analysed in Kubicki et al. (2016). To avoid local meteorological influences in the analyzed records, we used Ez data obtained only under so called "fair weather" conditions which request the absence of rain, snow, fog, low clouds, and wind velocity more than $6 \mathrm{~m} / \mathrm{s}$.

Atmospheric electricity observations from Swider and Hornsund have provided many cases of magnetic storm and substorm effects, as described below. We term the Ez deviations "positive" if the Ez magnitude increases and "negative" ones if the Ez magnitude decreases.

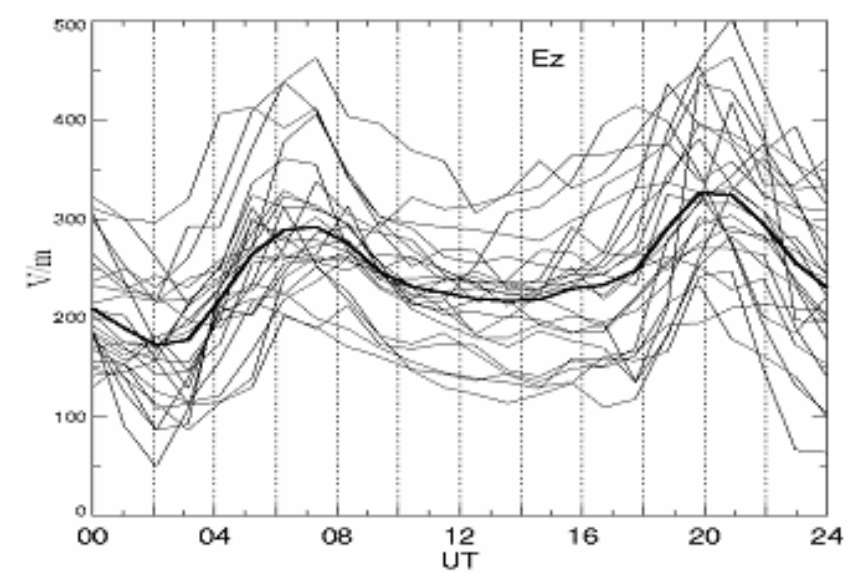

Fig. 3. The daily variations of the "fair weather" Ez(quiet) values at Swider observed under quiet geomagnetic conditions (Kpindex $<2$ ) over 22 selected days in 2000 , the solid curve shows the mean values.

\section{Middle latitudes.}

Land observation stations, such as Swider, especially in highly-populated areas often suffer from high and variable pollution (aerosols), the effects of which shift the local atmospheric field from its global diurnal pattern. The air turbulence which drives atmospheric convection currents also could affect the electric field and makes Ez monitoring more difficult.

To get rid of influence of strong geomagnetic disturbances on Ez variations, we plotted about 20 events of the Ez(quiet) values measured under the quiet geomagnetic conditions (Kp index <2) in Fig. 3. The significant scatter within a range $200-450 \mathrm{~V} / \mathrm{m}$ is seen respect to the Ez mean values. However, the main tendency in the daily Ez variation is apparent: minimum is near 01-04 UT and maximum - near 18-22 UT like the Carnegie curve (Fig.2) behavior. The hourly mean Ez level (Fig. 4) is much higher in winter than in summer (Kubicki et al., 2014). Thus, even under the quiet geomagnetic conditions, the measured on-situ "fair weather" Ez values may drastically change in time, so, it is not correct to use the Ez daily means as a basic level of a given day reference as it has been done previously in many papers. In contrast, to assess the influence of geomagnetic disturbances on Ez, we use the traditional geomagnetic method: to compare a given disturbed day with the nearest magnetically quiet period.

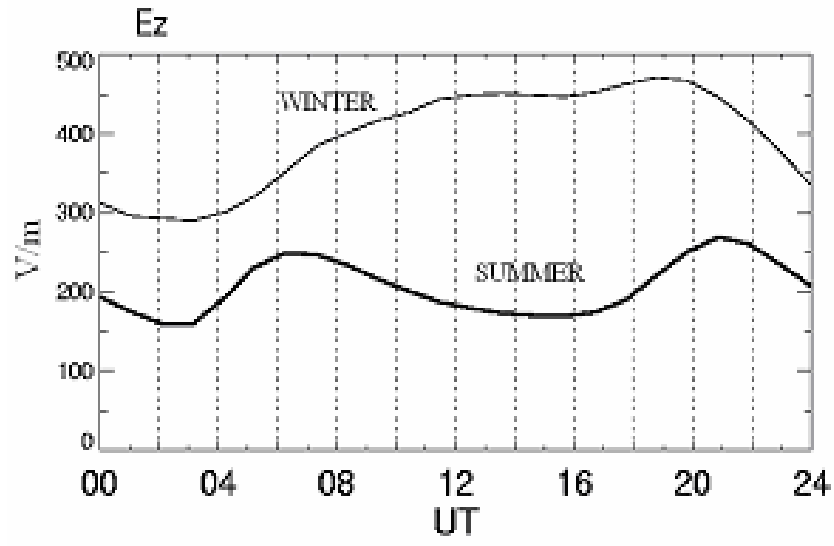

Fig. 4. The daily variations of the hourly mean Ez values in "fair weather" days in winter ( 89 days) and summer (289 days) at Swider from 1965 to 2000 (after Kubicki et al. 2014).

It is well know that the largest magnetospheric disturbances are happened in the main phase of magnetic storms and represented strong magnetic substorms at night side of the Earth. We found (Kleimenova et al. 2008, 2013) that the strongest effects of magnetic storm in mid-latitude Ez variations were observed not during the night but in the daytime.

Under the "fair weather" conditions in Swider in 2000-2004, we selected 14 magnetic storms. Our analysis showed that in 11 of 14 events of magnetic storms, a noticeable effect was observed, namely, we found the lower storm-time Ez values relatively to the non-storm period. The daytime negative Ez deviations within the time scale of 1-3 hours have been recorded simultaneously with the occurrence of the magnetic substorm at the night side of the Earth.

As example, the Ez effects of two magnetic storms (on October 13-14, 2000 and May 23-24, 2000) are shown in Fig. 5. As a magnetic storm characteristic we used the Dst-index (Gonzales et al. 1994) plotted in Fig 5a. The magnetic storm of October 13-14, 2000 stated on 13 October at 00 UT and was two-steps storm with two main phases (shown by red horizontal lines). The day before storm (12 October) was magnetically quiet ( $\mathrm{Kp}$ index $=1)$. The Ez(quiet) variations during this day are shown by the thin curve. The Ez values during this storm were lower than the Ez(quiet), except 10-14 UT on 13 October when the meteorological conditions were not good. Two significant negative bay-like Ez deviations were observed in the daytime on 14 October in association with the magnetic substorms observed at College (CMO) station, located at this time on the night side of the Earth. There was no local magnetic activity at Belsk (BEL) and Sodankyla (SOD) stations (Fig 5d) located near Swider meridian.

The similar Ez behavior was observed during the main phase of the magnetic storm on 23-24 May 2000 (Fig.5, right panels) started at 02 UT on 24 May. The meteorological conditions in the night-early morning time were not good, and the strong negative bay-like Ez deviation was clearly seen near noon in association with night-side magnetic substorm onset at College (CMO) station (marked by vertical red line). As the Ez(quiet) we applied the Ez variations on the previous day (23 May) because in this day, the Dst-index was near zero. 
a)
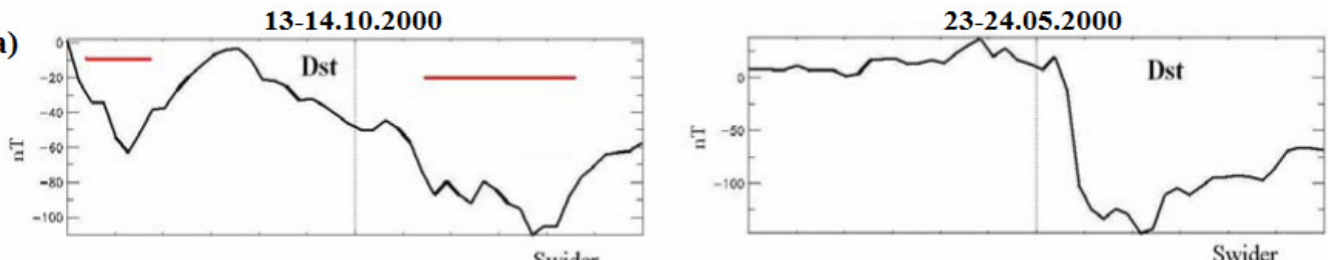

b)
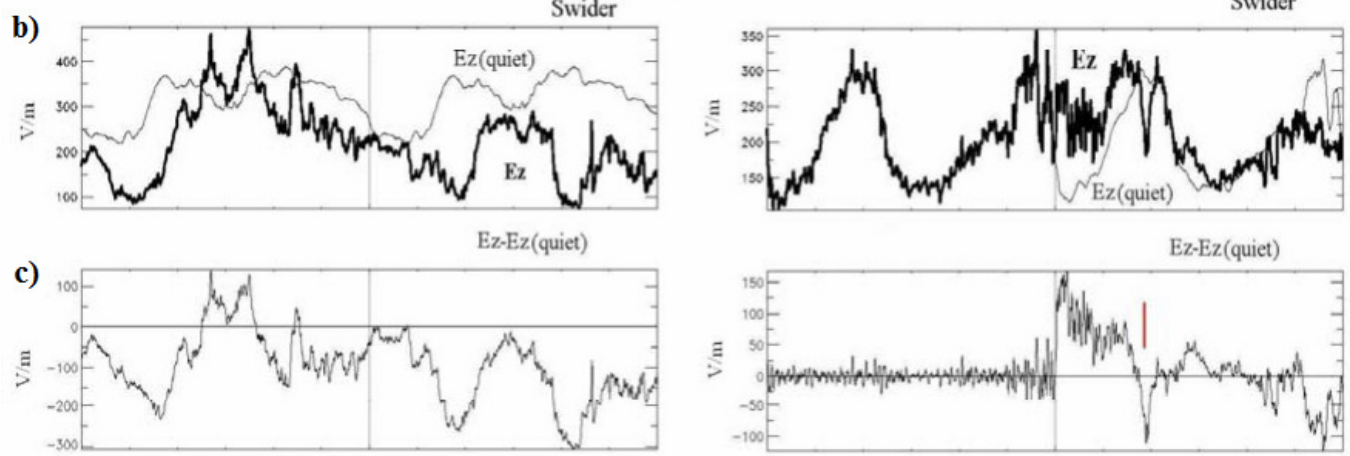

d)
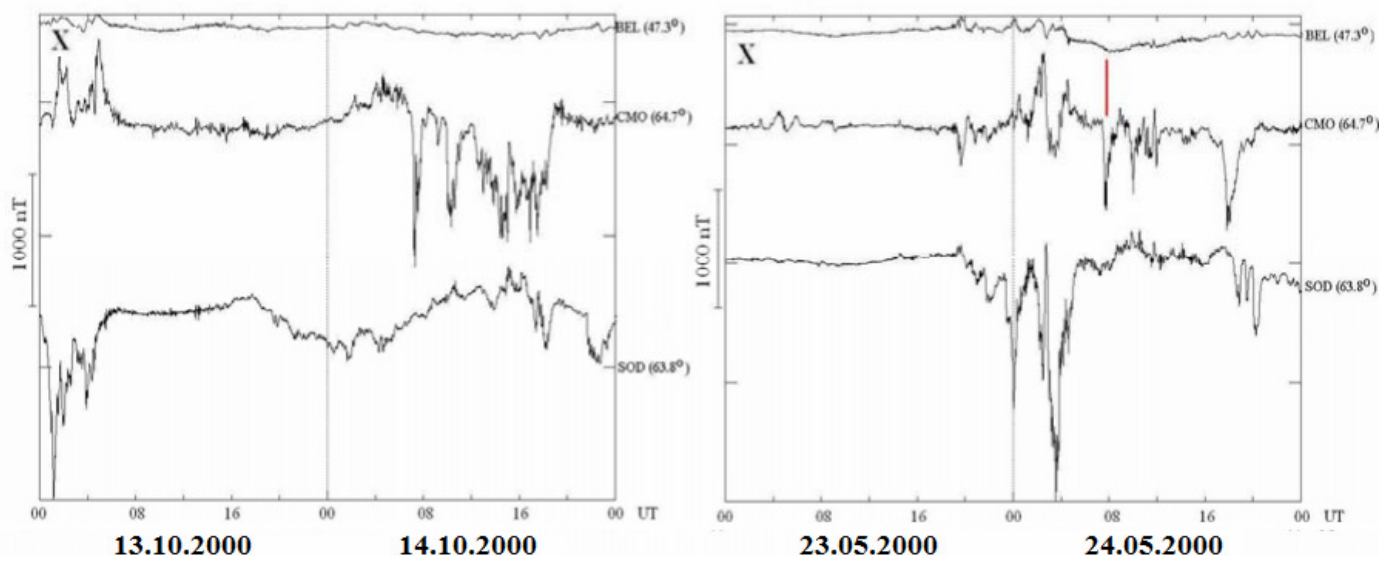

Fig. 5. a) Dst-variations; b) The storm-time Ez (thick curve) and the quiet-time Ez (thin curve); c) The calculated difference between these values; d) The magnetograms from BEL (near Swider), and auroral latitude CMO (night-side), and SOD (day-side) stations, after Kleimenova N.G. et al. (2008)
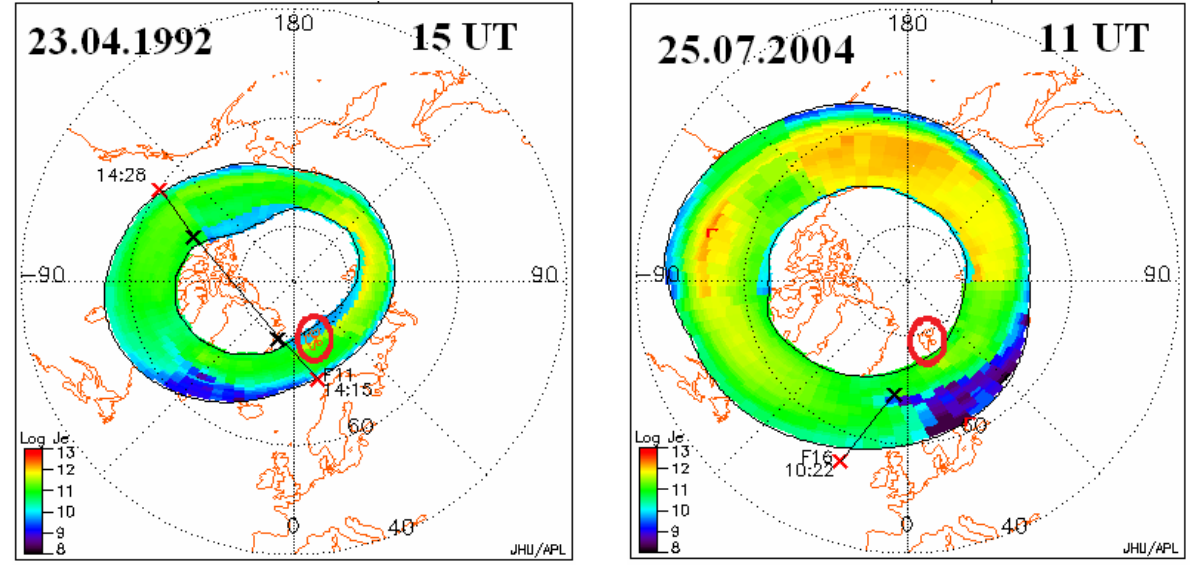

Fig. 6. The location of the Hornsund station (marked by a small red circle) and the auroral oval position accordings to OVATION model (for the details see the text).

We would like to note that in considered two storm events as well as many other storms, it was not necessary to compare the given Ez measurements with the the Ez(quiet) values. The effect was significant and could be seen even in the raw observation data.

The similar storm-time Ez effects, namely, occurrence daytime negative bay-like Ez deviations at Swider accompanied by a magnetic substorm onset at the night-side (College), were typical for all considered magnetic storms. We suppose that the Ez deviations could be associated with the modification of the ionospheric conductivity increasing due to night-side substorm energetic electron precipitation. Another plausible reason could be the interplanetary electric field penetrating into the magnetosphere during substorm.

Magnetic storms and substorms, caused by solar wind and interplanetary magnetic field disturbances of the Earth's magnetosphere, could influence the global 


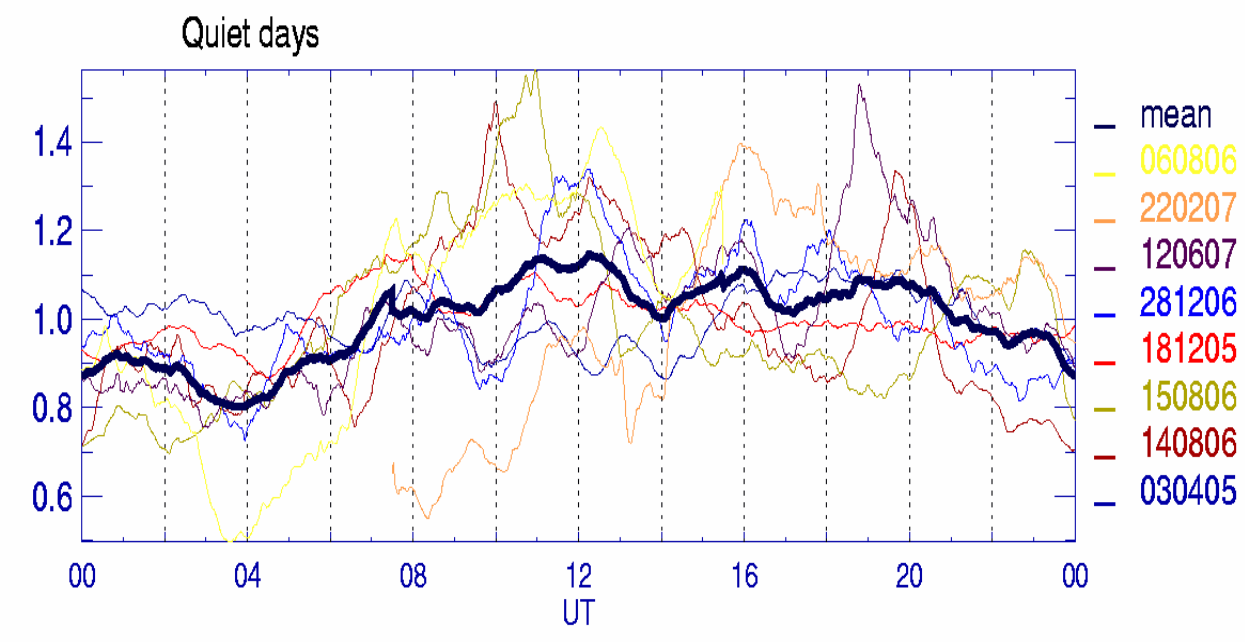

Fig. 7. The daily variations of the "fair weather" Ez(quiet) values at Horsund observed under quiet geomagnetic conditions (Kp-index <2) over several selected days in 2005-2007, the solid curve shows the mean values.

electric circuit state via changing of ionospheric conductivity due to particle precipitation or modification of cosmic ray flux, or via the direct interplanetary electric field penetration into the magnetosphere, and mapping to the ionosphere and the ionospheric convection changes. Thus, the variations in the atmospheric electricity represent one of the final stage the solar wind-magnetosphereatmosphere coupling.

\section{Polar latitudes.}

Depending on the level of geomagnetic activity, Hornsund station could be mapped within the auroral oval or in the polar cap in the vicinity of the auroral oval boundary, as it is shown in Fig. 6 (the Horsund location is marked by a small red circle). This Figure demonstrates the auroral oval position according to OVATION model (http://sd-www.jhuapl.edu/Aurora/ovation) based on energetic particle monitoring by the polar-orbiting DMSP satellites (in this event Fll and Fl6) and the solar wind and Interplanetary Magnetic Field measurements by ASE spacecraft.

The number of the "fair weather" days at the polar Arctic region (Hornsund station) was small, on average not more 3-4 days per month. The daily Ez variations are not as expressive as at low latitudes and did not show significant minima and maxima like the Carnegie curve. The diurnal "fair weather" Ez variations at Hornsund, normalized by the corresponding daily mean value, are plotted in Fig. 7 for the several magnetically quiet days in 2005-2007. It is seen that the different day scattering is much stronger than the mean Ez daily variations.

At the polar region, the interaction of the solar wind with the Earth's magnetic field leads to the polar convection driven by the horizontal electric field across the polar cap directed dawn-to-dusk. For structures larger than $\sim 500 \mathrm{~km}$, this polar cap potential can produce significant vertical electric fields at ground level in the polar cap [e.g., Tinsley, 2000]. In the auroral oval, the strongest effects in atmospheric electricity could be associated with magnetic substorms [Sao,
1967; Olson, 1971; Bandilet et al., 1986; Belova et al., 2000].

Substorm potential effects in Ez variations at the polar station Hornsund have been analysed in (Michnowski et al. 1991, 1996, 2007; Nikiforova et al., 2003, 2005; Kleimenova et al. 2010, 2011, 2017). As a rule, at this time, the Hornsund station was mapped inside the auroral oval in the vicinity of its polar boundary. It is well known that substorms occur inside the auroral oval in the local night, from the evening to the morning [Akasofu, 1964] and accompanied by an enhancement of the polar ionospheric convection. The listed above papers demonstrated that the substorm effects in the high-latitude Ez variations had different sign of deviations depending on the local time. It was found that the effects were "positive" in the local morning and "negative" in the evening.

This result has been confirmed by the Ez measurements at Horsund during 20 substorms in which the Ez effects were seen. These substorms have been selected at the Scandinavian magnetometer chain IMAGE in 2004-2006 under the "fair weather" intervals at Horsund: there were 12 substorms happened in the local morning and 8 events in the evening. It was not statistical analysis. Here we would like only to pay attention to the possible substorm effects on Ez measurements. In the future, we plan to conduct a detailed study of the types of high latitude substorms (polar or extended) demonstrating the greatest impact on Ez as well as the features of substorms that do not affect Ez.

Two examples of the substorm effects in the morning and evening Ez variations at Horsund are shown in Fig. 8a. The evening events are marked by the blue arrows and the morning events by the red ones. The corresponding maps of the polar ionospheric plasma convection are presented in Fig. $8 \mathrm{~b}$ (the maps were taken from SuperDARN radar data http://vt.superdarn.org/). It is seen that in the case of a morning (i.e. "positive") Ez effect, Horsund was mapped inside a "positive" ionosphere convection vortex (pink color), and of the evening - inside a "negative" one (blue color). Thus, we could speculate that the sign of Ez excursion controlled by the station 

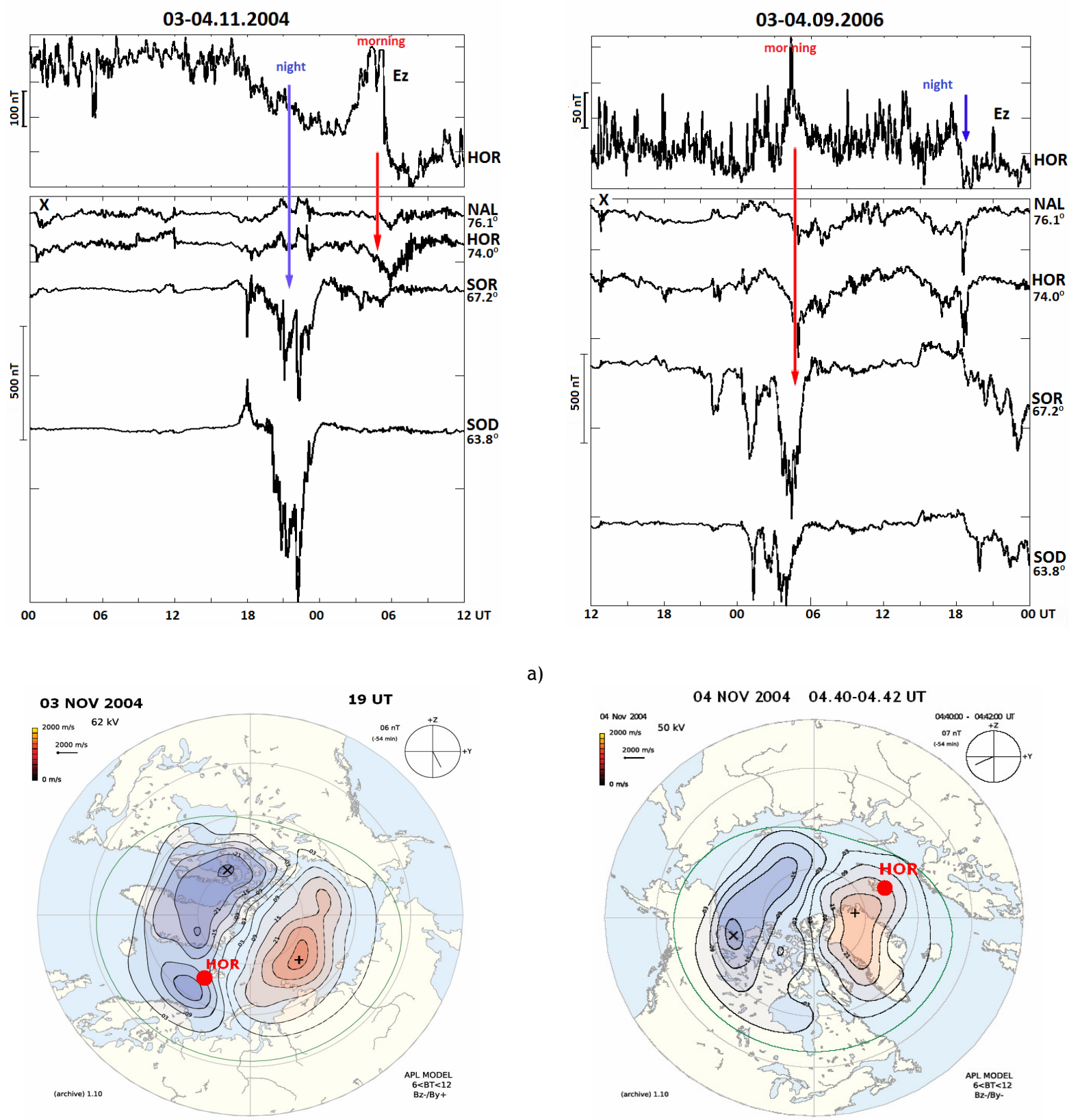

a)

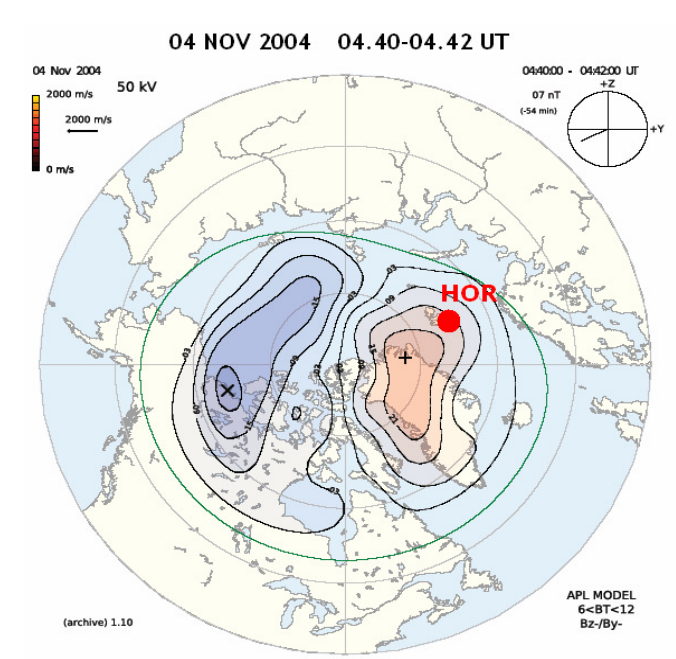

b)

Fig. 8. a) - Two examples of the negative Ez deviation during the night substorms and the positive Ez deviation during the morning substorms (after Kleimenova et al., 2010), b) - the polar ionosphere convection maps in the substorm correspondent time

location relatively to the position of the "positive" or "negative" vortex of the polar ionosphere plasma convection.

Thus, ground-based Ez observation data could be one of the sensible sensor of interactions in the solar wind-magnetosphere-ionosphere-atmosphere system.

\section{Summary}

1. The global atmospheric electric circuit state is controlled not only by the world thunderstorm activity but by magnetosphere-ionosphere disturbances (space weather conditions) as well.

2. The effect of the magnetic substorms during a magnetic storm main phase was established for the first time in the mid-latitude atmospheric electricity (Ez). The strong daytime Ez negative anomalies were revealed in association with night-side magnetosphere substorm onsets under any local magnetic activity

3. Substorm related Ez effects were observed at high-latitudes as well. It was found that the polar latitude Ez deviations could be associated with simultaneous substorms at auroral latitudes and demonstrated "positive" deviations in the local morning and "negative" ones in the evening correspondently to the station location in the "positive" or "negative" vortex of the polar ionospheric plasma convection.

\section{Acknowledgements}

This work was partly supported by the statutory activities in frame of the IGF PAS grant No 3841/E$41 / 5 / 2017$ of the Ministry of Science and Higher Education of Poland. 


\section{References}

Akasofu, S.-I.: 1964, Planet Space Sci. 12 (4), 273.

Bandilet, O.I., Kanonidi, Kh.D., Chernisheva, S.P., and Sheftel', V.M.: 1986, Geomag. Aeron, 26(1), 159.

Belova, E., Kirkwood, S., and Tammet, H.: 2000, Ann. Geophys., 18(12), 1623.

Berlinski, J., Pankanin, G., and Kubicki, M.: 2007: in Proc. 13th Intern. Conf. on Atmospheric Electricity, Beijing, China, I, 124.

Frank-Kamenetsky, A.V., Troshichev, O.A., Burns, G.B., and Papitashvili, V.O.: 2001, J. Geophys. Res., 106, 179.

Frank-Kamenetskii, A.V., Kotikov, A.L., Kruglov, A.A., Burns, G.B. Kleimenova, N.G., et al: 2012, Geomag. Aeron. 52(5), 629.

Gonzales, W.D., Joselyn, J.A., Kamide, Y., Kroehl, H. W., Rostoker, G., Tsurutani, B.T., and Vasyliunas, V.M.: 1994, J. Geophys. Res., 99, 5771.

Kleimenova, N.G., Kozyreva, O.V. Michnowski, S., and Kubicki, M.: 2008, Geomag. Aeron. 48(5), 622.

Kleimenova, N.G., Kozyreva, O.V., Kubicki, M. and Michnowski, S.: 2010, Geomag. Aeron. 50(1), 48.

Kleimenova, N.G., Kozyreva, O.V., Kubicki, M. and Michnowski, S.: 2011, Geomag. Aeron. 51(3), 394.

Kleimenova, N., Kozyreva, O., Michnowski, S., and Kubicki, M.: 2013, J. Atmos. Solar-Terr. Phys. 99, 117.

Kleimenova N. G., Kubickib, M., Odzimek, A., Malysheva, L.M., and Gromova, L.I.: 2017, Geomag. Aeron., 57 (3), 266.

Kubicki M.: 2001, Publ. Inst. Geophys. Pol. Acad. Sci., D-56(333), 3.

Kubicki, M., Odzimek, A., Kleimenova, N.G., Kozyreva, O.V., Neska, M.: 2014, in Proc. 15th Intern. Conf. Atmospheric Electricity, Oklahoma, U.S.A., 9 pp.

Kubicki M., Odzimek A., and Neska, M.: 2016, Atmos. Res. 178179, 329.

Michnowski, S., Szymanski, A., Nikiforova, N.N., Kozyreva O.V., Ermolenko, D., Zielkowski, K.: 1991, Publ. Inst. Geophys. Pol. Acad. Sci., D-35(238), 83.

Michnowski, S., Nikiforova, N.N., and Kleimenova, N.G.: 1996, in Proc. 10th Intern. Conf. on Atmospheric Electricity, Osaka, Japan, 520.

Michnowski, S.: 1998, J. Geophys. Res., 103, D12, 13939.

Michnowski, S., Kubicki, M., Kleimenova, N., Nikiforova, N., Kozyreva, O., and Israelsson, S.: 2007, in Proc. 13th Intern. Conf. on Atmospheric Electricity, Beijing, China, 1, 4.

Michnowski, S., Odzimek, A., Kleimenova, N.G., Kozyreva, O.V., Kubicki, M. and Nikiforova, N.N.: 2014, in Proc. 15th Intern. Conf. on Atmospheric Electricity, Oklahoma, U.S.A., 8 pp.

Nikiforova, N.N., Kleimenova, N.G., Kozyreva, O.V., Kubicki, M., and Michnowski, S.: 2003, Geomag. Aeron., 43(4), 29.

Nikiforova, N.N., Kleimenova, N.G., Kozyreva, O.V., Kubicki, M. and Michnowski, S.: 2005, Geomag. Aeron., 45(1), 148.

Odzimek, A., and Lester, M.: 2009, Publs. Inst. Geophys. Pol. Acad. Sc., D-73(412), 37.

Odzimek, A., Kubicki, M., Lester, M. and Grocott, A.: 2011, in Proc. 14th Intern. Conf. on Atmospheric Electricity, Rio de Janeiro, Brazil, 4 pp.

Olson, D.E.: 1971, Pure Appl. Geophys. 84, 118.

Parkinson, W.C. and Torreson, O.:1931, Union Terr. Magn. Electr. Bull., 8, 340 .

Roble, R.G.: 1985, J. Geophys. Res., 90 (4D), 2156.

Rycroft, M.J., and Harrison, R.G.: 2011, Space Sci. Rev., 168, 363.

Rycroft, M.J., Nicoll, K.A., Aplin, K.L., and Harrison, R.G.: 2012, J. Atmos. Sol-Terr. Phys. 90-91, 198.
doi.org/10.1016/j.jastp.2012.03.015.

Sao, K.: 1967, J. Atmos. Terr. Phys., 29, 213.

Tinsley, B.A.: 2000, Space Sci. Rev. 94 (1-2), 231.

Tinsley, B.A.: 2008, Rep. Prog. Phys. 71, 066801.

Tinsley, B. A. and Zhou. L.: 2006, J. Geophys. Res. 111, D16205.

Wilson, C.T.R.: 1920, Phil. Trans. Roy Soc. London A. 221, 73.

Williams, E.R.: 2009, Atmos. Res. 91( 2-4), 140.

Williams, E., and Mareev, E.: 2014, Atmos. Res. 135-136, 208.

Zhdanov, R.F., Moiseev, V.G., et al.: 1984, Geomag. Aeron., 1984, 24(5), 844. 